\title{
Predictors of Time to Discharge in Patients Hospitalized for Behavioral and Psychological Symptoms of Dementia
}

\author{
Tatsuru Kitamura Maki Kitamura Shoryoku Hino Koichi Kurata \\ Department of Neuropsychiatry, Ishikawa Prefectural Takamatsu Hospital, Kahoku City, Japan
}

\section{Key Words}

Dementia - Behavioral disorders · Psychiatric symptoms · Length of hospitalization ·

Predictors · Patient care management

\begin{abstract}
Background/Aims: In Japan, more than 50,000 patients with dementia are housed in psychiatric facilities, a trend precipitated by prolonged hospitalizations. This study aimed to determine predictors for the time to discharge in patients hospitalized for behavioral and psychological symptoms of dementia (BPSD). Methods: Medical charts of patients admitted to an acute psychogeriatric ward for treatment of BPSD were reviewed. Cox's proportional hazards model was used to evaluate relationships between active behavioral problems and/or demographics at the time of admission, and the time until favorable discharge (FD), defined as discharge to the patient's own home or a care facility. Results: For the 402 study patients included in this study, median time to FD was 101 days. In addition to family and residential factors, multivariate analysis identified higher Mini-Mental State Examination scores as independent clinical predictors for a shorter hospital stay, whereas male gender and combative behavior as the primary reason for hospital admission were predictors for a longer hospital stay. Conclusion: Clinical characteristics can be predictive of the time to discharge for patients with BPSD. Earlier interventions and enhanced care strategies may be needed for patients with a lower likelihood of FD.

Copyright $(2013$ S. Karger AG, Basel
\end{abstract}

\section{Introduction}

The Japanese government launched the long-term care insurance strategy in 2000 as a measure to meet the impending increase in the number of patients with dementia $[1,2]$. As part of this policy, a broad network of in-home care services was established [3-5] in addition 
Kitamura et al.: Predictors of Time to Discharge in Patients Hospitalized for Behavioral and Psychological Symptoms of Dementia

to various community-based care facilities to support patients with dementia to live independently within the community and outside the hospital system. Despite these service provisions, patients often require hospitalization in a psychiatric facility. The primary reason for hospital admissions is worsening behavioral and psychological symptoms of dementia (BPSD), which manifest in 90\% of dementia patients [6].

The goal of in-patient treatment for BPSD is the prompt remission of symptoms and to return patients to their communities as soon as possible. Recently, however, there has been an increase in prolonged hospital stays. Even after remission of BPSD, some patients experience difficulties being accepted by their communities following their discharge from a psychiatric hospital. In 2010, the Ministry of Health, Labour and Welfare reported that the average length of a hospital stay for dementia patients admitted to psychiatric hospitals was 944.3 days [7]. Consequently, the number of patients housed in psychiatric hospitals continues to rise, and is currently greater than 50,000 across Japan [8]. During the lengthy course of their hospital stay, some patients die, whereas others are transferred to medical hospitals because of their deteriorating physical condition. These outcomes are considered to constitute 'unfavorable discharges' (UFDs). In this context, a 'favorable discharge' (FD) encompasses moving patients to their own home or to a community-based care facility.

In addition to family and/or residential circumstances, a patient's clinical characteristics likely contribute to the time until discharge. However, to our knowledge, no studies have investigated the exact factors contributing to time until discharge.

The aim of the present study was to determine the factors predicting the time to FD in patients hospitalized for the treatment of BPSD. The time until FD was examined in relation to patient profiles at the time of their admission, with a particular emphasis on possible clinical predictors.

\section{Methods}

The medical records of patients hospitalized in the acute psychogeriatric ward of Ishikawa Prefectural Takamatsu Hospital were reviewed. The retrospective analysis was approved by the institutional review board, which also waived the requirement for written informed consent.

\section{Patients}

Consecutive patients admitted to the acute psychogeriatric ward at Ishikawa Prefectural Takamatsu Hospital for the treatment of BPSD between April 2006 and November 2009 were enrolled in the study. All patients had severe BPSD such that they could not be cared for in their own home or care facility, or be treated in an out-patient setting. Patients with severe physical comorbid diseases were deemed ineligible for hospitalization in the acute psychogeriatric ward; instead they could receive prioritized medical treatment for their physical comorbidities. Patients with a record of past hospitalization in our acute psychogeriatric ward and who had behavioral symptoms prior to their cognitive decline or psychiatric comorbidities were excluded from the study.

\section{Measures}

In all patients, the following items were evaluated and recorded within 1 week of admission according to the institutional protocol.

Demographics. Information was obtained for each patient regarding gender, age, living situation before hospitalization (including the type of residence and family situation), and relationship with the primary caregiver from patient interviews or responses to question- 
naires administered to family members or staff at the care facility/hospital by a psychiatric social worker. Residency prior to hospitalization was classified as living in his/her own home, a group home, care facility, or medical hospital. In Japan, group homes are care facilities in which groups of people spend their daily lives basically on their own, with staff support only when needed. In the present study, 'family situation' referred to the people with whom the patient had lived prior to hospitalization. The family situation of patients living with family members other than their partner or with staff in care facilities, group homes, or medical hospitals was classified as 'other'. The relationships between the patient and primary caregiver were categorized as partner, son or daughter, other family member or relative, or staff.

Reasons for Admission. Behavioral problems causing distress for the caregiver, which had become the primary reason for hospitalization, were recorded on the basis of interviews conducted with the caregiver. The reasons for hospitalization were classified as combative behavior, overactivity, or apathy or depression. Combative behavior comprised physically or verbally aggressive behavior during or between care provisions, such as hitting, kicking, biting, throwing things, cursing, and screaming. Overactivity included nonaggressive behavior that required constant monitoring, such as aimless wandering, trying to reach a different place, restlessness, or repetitive actions/mannerisms. Apathy and depression included serious apathetic or depressive behavior, such as severe loss of appetite, refusal to eat, refusal to take medication, or suicidal tendencies.

Type of Dementia. A diagnosis of dementia was made by either of the two experienced geriatric psychiatrists (T.K. and M.K.) according to DSM-IV criteria following interviews with patients and family members or staff, as well as on the basis of physical and neurological findings, laboratory data, and brain imaging studies.

Behavioral and Psychiatric Symptoms. The prevalence of behavioral and psychiatric symptoms was evaluated by two geriatric psychiatrists (T.K. and M.K.) using the Behavioral Pathology in Alzheimer's Disease (BEHAVE-AD) rating scale [9]. The presence or absence of symptoms in each of seven clusters comprising a subscale of BEHAVE-AD was recorded, including paranoid and delusional ideation, hallucinations, aggressiveness, activity disturbances, diurnal rhythm disturbances, affective disturbances, and anxieties or phobias.

Cognitive Function. Cognitive function was evaluated by geriatric psychiatrists in the acute psychogeriatric ward using the Mini-Mental State Examination (MMSE) [10].

Functional State of Daily Living. Activities of daily living (ADL) were scored by welltrained nursing staff according to the Nishimura-style senile ADL (N-ADL) scale [11], which is one of the most commonly used scales for the evaluation of ADL in Japan. The N-ADL scale evaluates five items: walking/sitting, range of activities, dressing/bathing, eating, and excretion. Each item is scored on a scale of $0-10$ points, with the total (maximum score 50) taken as the N-ADL score. Nursing staff rated the reliability of the scale, when completed, as good.

\section{Interventions}

Patients received pharmacological interventions as indicated clinically. All patients were treated under the supervision of the two geriatric psychiatrists (T.K. and M.K.) based on their clinical judgment. There was no limit to the time allowed on the acute psychogeriatric ward.

\section{Statistical Analysis}

The primary endpoint of the present study was time to FD, defined as moving the patient to his/her own home, a group home, or another care facility. UFDs were defined as death during hospitalization or patient transfer to a medical hospital because of their deteriorating physical condition [12]. 
Kitamura et al.: Predictors of Time to Discharge in Patients Hospitalized for Behavioral and Psychological Symptoms of Dementia

Data management and statistical calculations were performed using Stata version 11.0 (Statacorp, College Station, Tex., USA). Differences between groups (FD vs. UFD) in terms of age, MMSE score, N-ADL score, and dose of antipsychotics used during hospitalization were evaluated using t tests. Differences in frequency were analyzed using the $\chi^{2}$ test.

Kaplan-Meier analysis was used to calculate estimates of hospital stay probability from time of admission until FD. UFDs or being hospitalized at the end of the study were treated as censors. Patients' demographic and clinical characteristics at the time of hospital admission were analyzed for their association with time to FD using univariate Cox's proportional hazards regression model. To determine independent predictors of time until discharge, we used a multivariate Cox's proportional hazards regression model. In this model, backward elimination was used with a cutoff of $\mathrm{p}=0.1$.

Classification and regression tree analysis (CART) of the failure time data was performed with the user-supplied Stata CART procedure by van Putten [13] to search for appropriate cutoff points to count covariates and assess the possibility of interactions among covariates. To develop a simple prediction tree, we used variables that were found to be significant in the multivariate Cox's proportional hazards regression model.

Two-tailed $\mathrm{p}<0.05$ was considered significant. For multiple comparisons, $\mathrm{p}$ values were adjusted using Bonferroni correction.

\section{Results}

Table 1 lists the demographic characteristics of the 402 patients identified as eligible for inclusion in the present study. Of these, 291 patients (72\%) were identified as having an FD (107 were discharged to their own home, 61 were discharged to a group home, and 123 were discharged to other care facilities), 108 patients (27\%) were identified as having a UFD (93 were transferred to another hospital and 15 died during hospitalization), and 3 patients remained in the acute psychogeriatric ward when the study ended. Analysis of the demographic characteristics revealed there were fewer men in the FD compared with UFD group, and that Alzheimer's disease was more frequent in the FD group. Furthermore, in the FD group, patients were more likely to have resided in their own homes and less likely to have resided in a medical hospital. Both MMSE and N-ADL scores were higher in patients in the FD group. Finally, the BPSD symptoms at admission in the FD group were less likely to be aggressiveness and diurnal rhythm disturbance.

Table 2 shows the psychotropic medications used during hospitalization. The daily dose of antipsychotics was higher in the UFD group, while a cholinesterase inhibitor was more frequently used in the FD group. The Kaplan-Meier survival curve (fig. 1) revealed that the median length of the hospital stay was 101 days (interquartile range 52-252).

Univariate analyses revealed that patients residing in their own home prior to hospitalization, higher N-ADL and MMSE scores at the time of admission, and apathy/depression as the reason for admission were significant predictors of a shorter hospital stay, whereas male gender, residing in a medical hospital prior to hospitalization, staff being the primary caregivers, combative behavior as the reason for admission, and BPSD symptoms of aggressiveness and diurnal rhythm disturbances at the time of admission were significant predictors of a longer hospital stay (table 3 ).

Multivariate analyses revealed that patients residing in their own home prior to hospitalization and higher MMSE scores were independent significant predictors of a shorter hospital stay, whereas male gender, living alone, having a son or daughter as the primary caregiver, and combative behavior as the reason for hospitalization were predictors of a longer hospital stay (table 4). There was a trend for older age to predict a shorter hospital stay. 
Table 1. Patient demographic characteristics

\begin{tabular}{|c|c|c|c|c|}
\hline & \multirow{2}{*}{$\begin{array}{l}\text { All patients } \\
(\mathrm{n}=402)\end{array}$} & \multicolumn{3}{|c|}{ Discharged patients } \\
\hline & & $\mathrm{FD}(\mathrm{n}=291)$ & UFD (n=108) & p value ${ }^{1}$ \\
\hline Men & $167(42)$ & $102(35)$ & $64(59)$ & 0.000 \\
\hline Age, years & $81.0 \pm 7.1$ & $81.0 \pm 7.0$ & $81.0 \pm 7.4$ & 0.927 \\
\hline Type of dementia & & & & 0.027 \\
\hline $\mathrm{AD}$ & $246(61)$ & $190(65)$ & $56(52)^{*}$ & \\
\hline $\mathrm{VaD}$ & $59(15)$ & $36(13)$ & $23(21)$ & \\
\hline Other & $97(24)$ & $65(22)$ & $29(27)$ & \\
\hline Residency before hospitalization & & & & 0.000 \\
\hline Own home & $232(58)$ & $183(63)$ & $48(44)^{* *}$ & \\
\hline Group home & $48(12)$ & $34(12)$ & $13(12)$ & \\
\hline Care facility & $42(10)$ & $34(12)$ & $8(7)$ & \\
\hline Medical hospital & $80(20)$ & $40(13)$ & $39(37)^{* * *}$ & \\
\hline Patient living & & & & 0.701 \\
\hline Alone & $83(21)$ & $59(20)$ & $23(21)$ & \\
\hline With partner only & $72(18)$ & $50(17)$ & $22(20)$ & \\
\hline With others & $247(61)$ & $182(63)$ & $63(59)$ & \\
\hline Caregiver relationship & & & & 0.093 \\
\hline Partner & 107 (27) & $73(25)$ & $34(31)$ & \\
\hline Son or daughter & $139(33)$ & $104(35)$ & $33(31)$ & \\
\hline Other family member/relative & 47 (14) & $40(14)$ & $7(7)$ & \\
\hline Staff & $109(27)$ & $74(26)$ & $34(31)$ & \\
\hline MMSE score & $9.4 \pm 7.9$ & $10.4 \pm 7.9$ & $6.5 \pm 7.2$ & 0.000 \\
\hline N-ADL score & $26.1 \pm 12.8$ & $28.8 \pm 12.0$ & $18.7 \pm 12.2$ & 0.000 \\
\hline Reason for admission & & & & 0.189 \\
\hline Combative behavior & $151(38)$ & $101(35)$ & $48(44)$ & \\
\hline Overactivity & $181(45)$ & $138(47)$ & $42(39)$ & \\
\hline Apathy or depression & $70(17)$ & $52(18)$ & $18(17)$ & \\
\hline \multicolumn{5}{|l|}{ BPSD } \\
\hline Paranoid and delusional ideation & $120(30)$ & $94(32)$ & $25(23)$ & 0.076 \\
\hline Hallucination & $82(20)$ & $65(22)$ & $17(16)$ & 0.143 \\
\hline Aggressiveness & $240(60)$ & $162(56)$ & $75(69)$ & 0.013 \\
\hline Activity disturbances & $371(92)$ & $269(92)$ & $99(92)$ & 0.798 \\
\hline Diurnal rhythm disturbances & $326(81)$ & $228(78)$ & $96(89)$ & 0.017 \\
\hline Affective disturbances & $165(41)$ & $124(43)$ & $40(37)$ & 0.315 \\
\hline Anxieties and phobias & $128(32)$ & $99(34)$ & $28(26)$ & 0.123 \\
\hline
\end{tabular}

Data are given as means \pm SD or as the number of patients in each group with percentages in parentheses, as appropriate. ${ }^{*} \mathrm{p}<0.05,{ }^{* *} \mathrm{p}<0.01,{ }^{* * *} \mathrm{p}<0.001$ compared with patients with an FD. AD $=$ Alzheimer's disease; $\mathrm{VaD}=$ vascular dementia. ${ }^{1}$ Dichotomous characteristics were compared using the $\chi^{2}$ test; continuous characteristics were analyzed using t tests.

Table 2. Psychotropic medications used during hospitalization

\begin{tabular}{lclll}
\hline Psychotropic medications & \multirow{2}{*}{$\begin{array}{l}\text { All patients } \\
(\mathrm{n}=402)\end{array}$} & \multicolumn{2}{l}{ Discharged patients } \\
\cline { 3 - 5 } & & $\mathrm{FD}(\mathrm{n}=291)$ & UFD (n=108) & $\mathrm{p} \mathrm{value}^{2}$ \\
\hline Dose of antipsychotics $^{1}, \mathrm{mg} / \mathrm{kg}$ & $3.8 \pm 4.2$ & $3.5 \pm 3.9$ & $4.6 \pm 5.0$ & 0.015 \\
Use of cholinesterase inhibitor & $166(41)$ & $134(47)$ & $29(27)$ & 0.001 \\
\hline
\end{tabular}

Data are given as means \pm SD or as the number of patients in each group with percentages in parentheses, as appropriate. ${ }^{1}$ Daily dose of antipsychotic in chlorpromazine equivalents employed at the maximum during hospitalization. ${ }^{2}$ Dichotomous and continuous characteristics were compared using the $\chi^{2}$ test and the $t$ test, respectively. 
Kitamura et al.: Predictors of Time to Discharge in Patients Hospitalized for Behavioral and Psychological Symptoms of Dementia

Fig. 1. Cumulative probability of hospital stay in patients with BPSD.

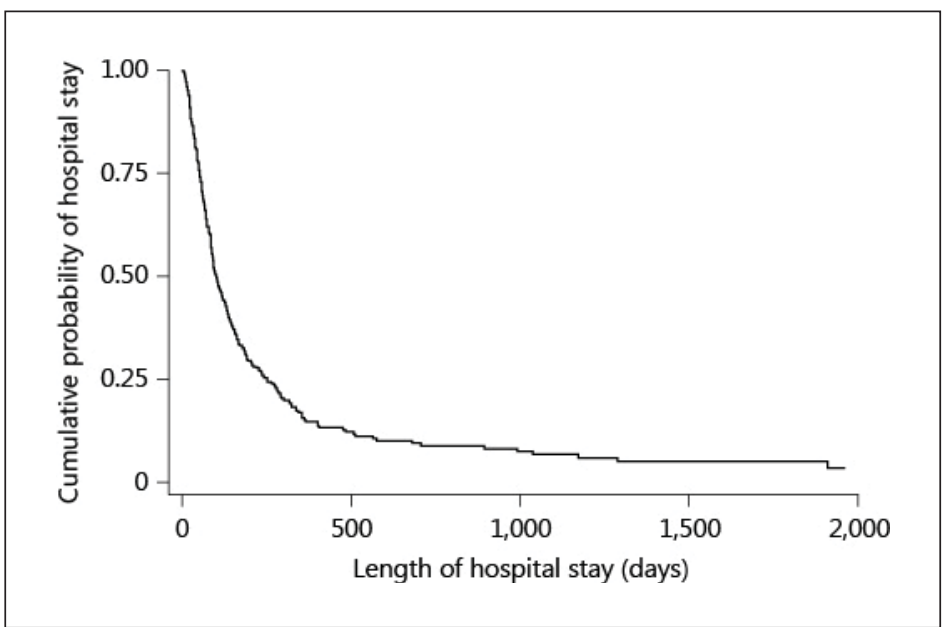

Table 3. Univariate Cox's proportional hazard regression for time to FD

\begin{tabular}{llll}
\hline Variable & Hazard ratio & $95 \% \mathrm{CI}$ & $\mathrm{p}$ value \\
\hline $\begin{array}{l}\text { Male gender } \\
\text { Type of dementia }\end{array}$ & 0.69 & $0.55-0.88$ & 0.003 \\
$\quad$ AD & 1.23 & $0.97-1.57$ & 0.091 \\
$\begin{array}{l}\text { Residency before hospitalization } \\
\quad \text { Own home }\end{array}$ & 1.43 & $1.12-1.81$ & 0.004 \\
$\quad$ Medical hospital & 0.69 & $0.50-0.97$ & 0.032 \\
Caregiver relationship & 0.75 & $0.58-0.98$ & 0.032 \\
$\quad$ Staff & 1.03 & $1.02-1.05$ & 0.000 \\
MMSE score & 1.02 & $1.00-1.02$ & 0.003 \\
N-ADL score & & & \\
Reason for admission & 0.68 & $0.53-0.87$ & 0.002 \\
$\quad$ Combative behavior & 1.38 & $1.02-1.87$ & 0.034 \\
$\quad$ Apathy or depression & & & \\
BPSD & 0.74 & $0.58-0.93$ & 0.010 \\
$\quad$ Aggressiveness & 0.69 & $0.45-1.07$ & 0.096 \\
$\quad$ Activity disturbance & 0.74 & $0.56-0.98$ & 0.037 \\
$\quad$ Diurnal rhythm disturbance & & \\
\hline$\quad$ Variables with p < 0.10 are listed. AD & Alzheimer's disease. & & \\
\hline
\end{tabular}

When building the CART, data were split into four groups with significantly different hazard ratios on the basis of MMSE score, combative behavior as the reason for admission, and male gender (fig. 2). According to the tree and the optimal cutoff point search, MMSE scores $>12$ predict the shortest hospital stay, whereas the combination of MMSE scores $\leq 12$ and combative behavior as the reason for hospitalization predicts the longest hospital stay.

\section{Discussion}

To our knowledge, the present study is the first to investigate the factors affecting the time to discharge among patients hospitalized for the treatment of BPSD. In addition to family and residential factors, multivariate analysis identified a further three clinical factors inde- 
Kitamura et al.: Predictors of Time to Discharge in Patients Hospitalized for Behavioral and Psychological Symptoms of Dementia

Fig. 2. CART analysis for time to FD. $\mathrm{n}=$ Number of observations; HR = hazard ratio

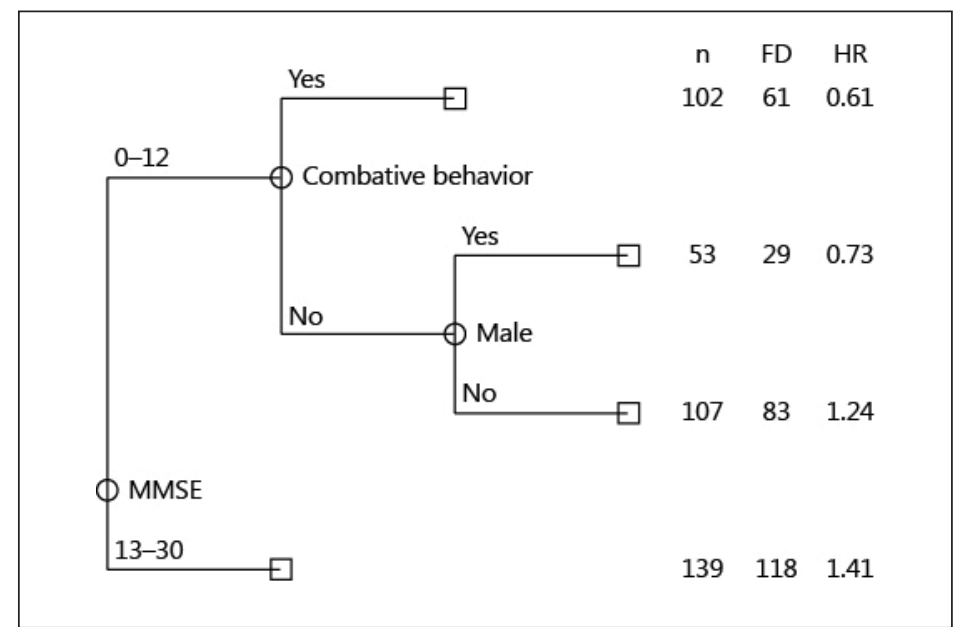

Table 4. Multivariate Cox's proportional hazard regression for time to FD

\begin{tabular}{llll}
\hline Variable & Hazard ratio & $95 \%$ CI & p value \\
\hline Male gender & 0.70 & $0.54-0.92$ & 0.010 \\
Age & 1.02 & $1.00-1.04$ & 0.065 \\
$\begin{array}{l}\text { Residency before hospitalization } \\
\quad \text { Own home }\end{array}$ & 1.34 & $1.04-1.73$ & 0.024 \\
$\begin{array}{l}\text { Patient living } \\
\quad \text { Alone }\end{array}$ & 0.64 & $0.46-0.88$ & 0.006 \\
$\quad \begin{array}{l}\text { Caregiver relationship } \\
\quad \text { Son or daughter }\end{array}$ & 0.73 & $0.54-0.97$ & 0.033 \\
$\quad \begin{array}{l}\text { MMSE score } \\
\text { Reason for admission }\end{array} \quad 1.03$ & $1.01-1.05$ & 0.000 \\
$\quad$ Combative behavior & 0.75 & $0.58-0.97$ & 0.026
\end{tabular}

Variables with $\mathrm{p}<0.10$ are listed. $\mathrm{AD}=$ Alzheimer's disease.

pendently associated with time to FD: MMSE score, combative behavior as the primary reason for admission, and male gender.

Cognitive function is strongly associated with performing instrumental ADL, which encompasses complex behaviors such as managing finances, handling medications, and housekeeping [14-16]. The care burden may be greater for patients with lower MMSE scores, and this could present a major obstacle to discharging patients after remission of BPSD. Contrary to our expectations, our analyses did not reveal N-ADL scores, which represent the performance of basic ADL, as independent predictors of time until discharge. In contrast with instrumental ADL, it has been reported that basic ADL are highly correlated with motor function and coordination [17, 18]. In Japan's long-term care insurance system, although eligibility is ranked according to assessments of both physical and cognitive status, higher care levels are provided for physical rather than cognitive impairments [19]. However, some studies $[20,21]$ have shown that the care burden for severely demented patients with mild physical disabilities could be considerably greater than that for patients with severe physical disabilities because demented patients may act without intention, which may necessitate constant supervision. 
Combative behavior refers to physically or verbally aggressive behavior. We have clearly shown in the present study that when aggressive behavior becomes prominent and is the primary reason for admission, it could prolong hospitalization. Although we did not evaluate chronological treatment response, our findings indicate that aggressive behavior may be less likely to respond to interventions, including pharmacotherapy, compared with other types of behavioral problems. Antipsychotics are currently the mainstay of treatment to control aggressive behavior; however, the available data regarding the efficacy of antipsychotics are inconsistent $[22,23]$ and the doses that can be used are limited because of the risk of considerable side effects [24] or death [25]. Safer alternatives [26, 27] include sedative/hypnotics, anticonvulsants, serotonergic agents, lithium, $\beta$-blockers, and estrogen, but there is insufficient evidence supporting the efficacy of these drugs.

Even after a patient's aggressive behavior is under control, there is likely to be an effect on the patient's interpersonal relationships [28], with caregivers likely to express disapproval of the patient's discharge because of the harmful nature of this sort of behavior [2931]. In addition to enhanced service provision, recently developed interventions targeting caregivers [32-35] may be beneficial in decreasing the burden and stress of caregiving. Trials have indicated that these measures can simultaneously improve the quality of life of both the patient and caregiver [36, 37].

In a previous study [12] on hospitalized patients with BPSD, we found that the profile of men at the time of admission was different to that of women: specifically, men were younger, more frequently had vascular dementia, were more frequently admitted from medical hospitals, had lower MMSE and N-ADL scores, were more frequently admitted because of combative behavior, and exhibited aggressiveness and diurnal rhythm disturbances on the BEHAVE-AD. These factors have been shown in the present study to be potentially associated with a longer period of hospitalization. In our previous study we also discussed the possible effect of inequality between genders in terms of the availability of care services. In Japan, 80\% of residents in care institutions are women [38] and there are relatively few beds specifically equipped for use by men. Furthermore, the majority of care workers are women [39], who often prefer that female residents are accepted into the care home because they are perceived to be easier to care for.

In the present study, CART analysis revealed that the aforementioned clinical predictors could be used to divide the data into four groups with different hazard ratios for FD. Patients with severely impaired cognitive function admitted primarily because of combative behavior had the lowest likelihood of FD. In patients exhibiting aggressiveness, pharmacological or nonpharmacological interventions involving both patients and caregivers may be required from the early stages of dementia before the symptoms become prominent. Factors related to family and residential circumstances were not used to split the data, and this suggests that these factors may act equally in each of the four groups to affect the likelihood of FD. The options following the discharge of patients admitted from care or medical facilities may be limited because these patients are usually in a worse physical condition and require constant formal care or supervision. In terms of relationships between caregivers and patients, it has been reported that children are less strongly committed to the caregiving relationship than spouses [40], and stronger support systems are needed for patients living alone. However, under the current long-term care insurance system in Japan, family composition is not supposed to be taken into account when deciding on the level of care required. Other social factors that may have an impact on the time to FD could include economic status or the availability of care resources.

There are some limitations to the present study that need to be acknowledged and addressed. As described above, because of the retrospective nature of this study based on a review of patients' medical charts, only limited information was available. In particular, we 
Kitamura et al.: Predictors of Time to Discharge in Patients Hospitalized for Behavioral and Psychological Symptoms of Dementia

did not evaluate caregiver burden, despite findings indicating its potential association with time to discharge [41]. Furthermore, although any pharmacotherapy was optimized to achieve remission of BPSD as soon as possible, our routine practice did not include chronological treatment response. These issues need to be taken into consideration in future studies, as well as in clinical practice. Finally, the present study was conducted in a single institution in a particular region of Japan. Future well-designed prospective and larger-scale studies are warranted to confirm our results.

In conclusion, clinical characteristics, such as lower MMSE scores, combative behavior as the primary reason for hospitalization, and male gender, could be predictors of a lower likelihood of FD in patients with BPSD. These findings should be taken into consideration when managing patients with BPSD to enable implementation of optimal treatment and care strategies to improve in-patient treatment outcomes.

\section{References}

1 Arai Y, Washio M, Miura H, Kudo K: Dementia care in Japan: insurance for long-term care legislation in Japan. Int J Geriatr Psychiatry 1998;13:572-573.

2 Nakane J, Farevaag M: Elder care in Japan. Perspectives 2004;28:17-24.

3 Campbell JC, Ikegami N: Long-term care insurance comes to Japan. Health Aff (Millwood) 2000;19:26-39.

-4 Traphagan JW: Independence, security, and the intergenerational social contract: home-helper services and elder care in rural Japan. Care Manag J 2003;4:216-222.

5 Traphagan JW: Culture and long-term care: the bath as social service in Japan. Care Manag J 2004;5:53-60.

-6 Finkel SI: Behavioral and psychologic symptoms of dementia. Clin Geriatr Med 2003;19:799-824.

7 Reports form the survey on dementia patients hospitalized in psychiatry hospitals (in Japanese). Japanese Ministry of Health, and Labour and Welfare. http://www.mhlw.go.jp/stf/shingi/2r9852000000z8ieatt/2r9852000000znwy.pdf.

8 Reports from the investigative commission on mental health and welfare in the future (in Japanese). Japanese Ministry of Health, and Labour and Welfare. http://www.mhlw.go.jp/shingi/2009/05/s0521-3.html.

-9 Reisberg B, Borenstein J, Salob SP, Ferris SH, Franssen E, Georgotas A: Behavioral symptoms in Alzheimer's disease: phenomenology and treatment. J Clin Psychiatry 1987;48(suppl):9-15.

10 Folstein MF, Folstein SE, McHugh PR: 'Mini-mental state'. A practical method for grading the cognitive state of patients for the clinician. J Psychiatr Res 1975;12:189-198.

11 Nishimura T, Kobayashi T, Hariguchi S, Takeda M, Fukunaga T, Inoue O, Kondo H, Niigawa H, Tanaka S, Yamashita M: Scales for mental state and daily living activities for the elderly: clinical behavioral scales for assessing demented patients. Int Psychogeriatr 1993;5:117-134.

12 Kitamura T, Kitamura M, Hino S, Tanaka N, Kurata K: Gender differences in clinical manifestations and outcomes among hospitalized patients with behavioral and psychological symptoms of dementia. J Clin Psychiatry 2012;73:1548-1554.

13 van Putten W: Classification and regression tree analysis with Stata. http://www.stata.com/meeting/2dutch/ cart.pdf.

14 Stern Y, Hesdorffer D, Sano M, Mayeux R: Measurement and prediction of functional capacity in Alzheimer's disease. Neurology 1990;40:8-14.

15 Bennett HP, Corbett AJ, Gaden S, Grayson DA, Kril JJ, Broe GA: Subcortical vascular disease and functional decline: a 6-year predictor study. J Am Geriatr Soc 2002;50:1969-1977.

16 Cahn-Weiner DA, Farias ST, Julian L, Harvey DJ, Kramer JH, Reed BR, Mungas D, Wetzel M, Chui H: Cognitive and neuroimaging predictors of instrumental activities of daily living. J Int Neuropsychol Soc 2007;13:747757.

17 Cahn DA, Sullivan EV, Shear PK, Pfefferbaum A, Heit G, Silverberg G: Differential contributions of cognitive and motor component processes to physical and instrumental activities of daily living in Parkinson's disease. Arch Clin Neuropsychol 1998;13:575-583.

-18 Boyle PA, Cohen RA, Paul R, Moser D, Gordon N: Cognitive and motor impairments predict functional declines in patients with vascular dementia. Int J Geriatr Psychiatry 2002;17:164-169.

19 Ito H, Miyamoto Y: Impact of long-term care insurance on institutional dementia care in Japan. Health Policy 2003;64:325-333.

20 Ito H, Tachimori H, Miyamoto Y, Morimura Y: Are the care levels of people with dementia correctly assessed for eligibility of the Japanese long-term care insurance? Int J Geriatr Psychiatry 2001;16:1078-1084.

-21 Miyamoto Y, Ito H, Otsuka T, Kurita H: Caregiver burden in mobile and non-mobile demented patients: a comparative study. Int J Geriatr Psychiatry 2002;17:765-773. 
22 Schneider LS, Pollock VE, Lyness SA: A metaanalysis of controlled trials of neuroleptic treatment in dementia. J Am Geriatr Soc 1990;38:553-563.

-23 Lonergan E, Luxenberg J, Colford J: Haloperidol for agitation in dementia. Cochrane Database Syst Rev 2002; 2:CD002852.

24 Wooltorton E: Risperidone (Risperdal): increased rate of cerebrovascular events in dementia trials. CMAJ 2002;167:1269-1270.

25 Schneider LS, Dagerman KS, Insel P: Risk of death with atypical antipsychotic drug treatment for dementia: meta-analysis of randomized placebo-controlled trials. JAMA 2005;294:1934-1943.

-26 Schneider LS, Sobin PB: Non-neuroleptic treatment of behavioral symptoms and agitation in Alzheimer's disease and other dementia. Psychopharmacol Bull 1992;28:71-79.

-27 Kunik ME, Yudofsky SC, Silver JM, Hales RE: Pharmacologic approach to management of agitation associated with dementia. J Clin Psychiatry 1994;55(suppl):13-17.

28 Tremont G, Davis JD, Bishop DS: Unique contribution of family functioning in caregivers of patients with mild to moderate dementia. Dement Geriatr Cogn Disord 2006;21:170-174.

29 Voyer P, Verreault R, Azizah GM, Desrosiers J, Champoux N, Bedard A: Prevalence of physical and verbal aggressive behaviours and associated factors among older adults in long-term care facilities. BMC Geriatr 2005;5:13.

30 Kunik ME, Walgama JP, Snow AL, Davila JA, Schulz PE, Steele AB, Morgan RO: Documentation, assessment, and treatment of aggression in patients with newly diagnosed dementia. Alzheimer Dis Assoc Disord 2007;21: $115-121$.

-31 Cohen-Mansfield J: Agitated behavior in persons with dementia: the relationship between type of behavior, its frequency, and its disruptiveness. J Psychiatr Res 2008;43:64-69.

32 Chan SW: Family caregiving in dementia: the Asian perspective of a global problem. Dement Geriatr Cogn Disord 2010;30:469-478.

33 Schulz R, Martire LM, Klinger JN: Evidence-based caregiver interventions in geriatric psychiatry. Psychiatr Clin North Am 2005;28:1007-1038, x.

-34 Belle SH, Burgio L, Burns R, Coon D, Czaja SJ, Gallagher-Thompson D, Gitlin LN, Klinger J, Koepke KM, Lee CC, Martindale-Adams J, Nichols L, Schulz R, Stahl S, Stevens A, Winter L, Zhang S, Resources for Enhancing Alzheimer's Caregiver Health (REACH) II Investigators: Enhancing the quality of life of dementia caregivers from different ethnic or racial groups: a randomized, controlled trial. Ann Intern Med 2006;145:727-738.

-35 Kunik ME, Snow AL, Davila JA, Steele AB, Balasubramanyam V, Doody RS, Schulz PE, Kalavar JS, Morgan RO: Causes of aggressive behavior in patients with dementia. J Clin Psychiatry 2010;71:1145-1152.

-36 Sorensen S, Pinquart M, Duberstein P: How effective are interventions with caregivers? An updated metaanalysis. Gerontologist 2002;42:356-372.

-37 Callahan CM, Boustani MA, Unverzagt FW, Austrom MG, Damush TM, Perkins AJ, Fultz BA, Hui SL, Counsell SR, Hendrie HC: Effectiveness of collaborative care for older adults with Alzheimer disease in primary care: a randomized controlled trial. JAMA 2006;295:2148-2157.

38 Current state of users of care facilities (in Japanese). Japanese Ministry of Health, Labour and Welfare. http:// www.mhlw.go.jp/toukei/saikin/hw/kaigo/service07/kekka4.html.

39 Current state of employees in care and welfare service (in Japanese). Japanese Ministry of Health, Labour and Welfare Website. http://www.mhlw.go.jp/shingi/2007/03/dl/s0329-12d.pdf.

40 Pot AM, Deeg DJ, Knipscheer CP: Institutionalization of demented elderly: the role of caregiver characteristics. Int J Geriatr Psychiatry 2001;16:273-280.

41 Arai Y, Kudo K, Hosokawa T, Washio M, Miura H, Hisamichi S: Reliability and validity of the Japanese version of the Zarit Caregiver Burden interview. Psychiatry Clin Neurosci 1997;51:281-287. 